\title{
Nonempirical hybrid functionals for band gaps and polaronic distortions in solids
}

\author{
Giacomo Miceli, ${ }^{1, *}$ Wei Chen, ${ }^{2}$ Igor Reshetnyak, ${ }^{1}$ and Alfredo Pasquarello ${ }^{1}$ \\ ${ }^{1}$ Chaire de Simulation à l'Echelle Atomique (CSEA), Ecole Polytechnique Fédérale de Lausanne (EPFL), CH-1015 Lausanne, Switzerland \\ ${ }^{2}$ Institute of Condensed Matter and Nanosciences (IMCN), Université Catholique de Louvain, Louvain-la-Neuve 1348, Belgium
}

(Received 17 August 2017; revised manuscript received 20 December 2017; published 26 March 2018)

\begin{abstract}
We construct hybrid functionals in a nonempirical way by fixing the fraction of Fock exchange through either the long-range screening or the generalized Koopmans' condition applied to defect charge-transition levels. These functionals not only give band gaps of solids as accurate as state-of-the-art $G W$ calculations, but are also capable of describing polaronic distortions. The Koopmans' condition is found to be satisfied across a series of defects, to the point of achieving accurate band gaps through a hydrogen probe. Extension to range-separated functionals demonstrates the robustness of the present approach.
\end{abstract}

DOI: 10.1103/PhysRevB.97.121112

Semilocal approximations to density functional theory (DFT) have long been the method of choice for the study of materials properties, due to the good compromise between accuracy and computational efficiency. However, one important deficiency of these schemes is the severe underestimation of the electronic band gap [1]. Another shortcoming involves the spurious interaction of an electron with its own charge density [2], which can more fundamentally be identified as the many-body self-interaction error $[3,4]$. The self-interaction error (SIE) manifests through the failure of semilocal DFT in describing electron localization associated with small polaron formation in wide-gap materials $[5,6]$. There are also indications that addressing the SIE deficiency might lead to an improved description of the band gap of solids [2,7-13], but the extent of this relationship has not clearly been delimited. The generally accepted electronic-structure method for overcoming the band-gap underestimation is the many-body $G W$ formulation [14-17]. However, this approach is computationally demanding and does not trivially allow for structural relaxation, as would be required for describing polaronic distortions.

There is therefore a general demand for having a more accessible scheme to correct for the shortcomings of semilocal DFT. Hybrid density functionals appear successful both in opening the band gap [18] and in describing polaronic distortions [19-21]. However, these functionals remain unsatisfactory insofar as they contain an undetermined fraction of Fock exchange $\alpha$ [22]. Range-separated hybrid functionals offer more flexibility but at the cost of even more parameters $[23,24]$. Two research directions have developed to fix such free parameters in a nonempirical fashion. The first direction leads to dielectric dependent hybrid functionals and consists of enforcing the long-range screening properties [25-27]. Based on the static Coulomb-hole screened-exchange approximation to the $G W$ self-energy [14], this can be achieved by setting $\alpha=1 / \varepsilon_{\infty}$ [18], where $\varepsilon_{\infty}$ is the high-frequency dielectric constant. This choice not only leads to improved band gaps

\footnotetext{
*giacomo.miceli@epfl.ch
}

$[18,28-35]$ and spectra [36,37], but also to a proper description of polaronic states [38]. In the second direction, the generalized Koopmans' condition is enforced [3]. Most applications focus on molecules [39-49] or on molecular crystals [50,51] and achieve an accurate description of the excitation spectrum $[52,53]$. However, the application of the same concept to extended systems has led to conflicting claims as far as the bandgap determination is concerned $[11,54]$. Nevertheless, hybrid functionals satisfying the generalized Koopmans' condition have generally been found to properly account for polaronic localization $[50,54,55]$.

In this Rapid Communication, we examine whether hybrid functionals, in which the free parameters are nonempirically set by enforcing physical constraints, can reproduce the experimental band gap of extended systems and concurrently account for polaronic distortions. To set a benchmark, we first focus on alkali halides, which show a wide spread of band gaps and the localization of polaronic holes. We perform state-of-the-art $G W$ calculations, which we use for assessing the accuracy by which the constructed hybrid functionals determine the band gap. The enforced physical constraints involve either the long-range screening or the generalized Koopmans' condition applied to defect levels. The dependence on the nature of the defect is also investigated and leads to the hydrogen interstitial as the probe of choice, which is then successfully applied to a wider range of materials. Allowing for higher flexibility as in range-separated functionals supports the robustness of the approach. The present results point to a close quantitative connection between the long-range screening, the SIE, and the band gap.

We first consider hybrid functionals $\operatorname{PBE} 0(\alpha)$, which contain a single parameter $\alpha$ corresponding to the fraction of Fock exchange [22]. Upon the creation of a point defect, the atomic structure is not allowed to relax, implying that the long-range screening is entirely described by $\varepsilon_{\infty}$. To overcome finite-size effects, the single-particle defect energy levels are corrected using the formula derived in Ref. [58], which is consistent with state-of-the-art total-energy corrections [59-61]. In this study, we only retain defect states that show well localized charge, as inferred from the delocalized screening charge 
TABLE I. Band gaps $E_{\mathrm{g}}$ obtained at the QS $G \tilde{W}$ level and highfrequency dielectric constants $\varepsilon_{\infty}$ calculated at the semilocal level for various alkali halides, compared with experimental values from Refs. [56] and [57], respectively. Mean absolute errors (MAEs) are given with respect to experiment.

\begin{tabular}{lllcc}
\hline \hline & $\varepsilon_{\infty}^{\text {theor }}$ & $\varepsilon_{\infty}^{\text {expt. }}$ & $E_{\mathrm{g}}^{\mathrm{QS} G \tilde{W}}(\mathrm{eV})$ & $E_{\mathrm{g}}^{\text {expt. }}(\mathrm{eV})$ \\
\hline $\mathrm{NaI}$ & 3.03 & 3.15 & 5.95 & 5.90 \\
$\mathrm{LiI}$ & 3.85 & 3.82 & 6.24 & 6.10 \\
$\mathrm{NaBr}$ & 2.56 & 2.70 & 7.18 & 7.10 \\
$\mathrm{LiBr}$ & 3.13 & 3.18 & 7.46 & 7.60 \\
$\mathrm{NaCl}$ & 2.22 & 2.38 & 8.27 & 8.97 \\
$\mathrm{LiCl}$ & 2.78 & 2.76 & 9.28 & 9.40 \\
$\mathrm{NaF}$ & 1.69 & 1.76 & 12.05 & 11.50 \\
$\mathrm{LiF}$ & 1.96 & 1.94 & 14.52 & 14.20 \\
$\mathrm{MAE}$ & 0.08 & & 0.26 & \\
\hline \hline
\end{tabular}

[60]. The dielectric constants $\varepsilon_{\infty}$ are determined within the random phase approximation at the semilocal level [62], and generally agree well with experiment, as shown for alkali halides in Table I. The band gaps used as reference are obtained with quasiparticle self-consistent $G W$ calculations $[63,64]$, in which effective vertex corrections are included in the screening $(\mathrm{QS} G \tilde{W})[17,65]$. We use the same setup as in the hybrid functional calculations, to enable meaningful comparisons between theoretical results. Computational details are given in the Supplemental Material [65]. Reference band gaps for alkali halides are reported in Table I and agree well with their experimental counterparts.

We first focus on various alkali halides, namely, NaI, LiI, $\mathrm{NaBr}, \mathrm{LiBr}, \mathrm{NaCl}, \mathrm{LiCl}, \mathrm{NaF}$, and LiF. These materials constitute a meaningful benchmark as they are dominated by long-range screening, exhibit localized polaronic hole states, and show a wide range of band gaps from 6 to $14 \mathrm{eV}$ (cf. Table I). To study the effect of incorporating the long-range screening in the functional $\operatorname{PBE} 0(\alpha)$, we set $\alpha=1 / \epsilon_{\infty}[18,33]$. The ensuing band gaps for the alkali halides are given in Table II and are compared to the QSG $\tilde{W}$ results in Fig. 1(a).

TABLE II. Band gaps (in eV) of alkali halides obtained using the hybrid functional PBE0 $(\alpha)$, in which the fraction of Fock exchange $\alpha$ (given in parentheses) is set through the long-range screening $\left(1 / \varepsilon_{\infty}\right)$, or through the enforcement of the Koopmans' condition on the $+/ 0$ transition of the polaron $\left(\alpha_{\text {polaron }}\right)$, or on the $+/ 0$ and $0 /-$ transitions of an interstitial $\mathrm{H}$ impurity $\left(\alpha_{\mathrm{H}(+/ 0)}\right.$ and $\left.\alpha_{\mathrm{H}(0 /-)}\right)$. Mean average errors are calculated with respect to QSG $\tilde{W}$ band gaps in Table I.

\begin{tabular}{lcccr}
\hline \hline & $1 / \varepsilon_{\infty}$ & $\alpha_{\text {polaron }}$ & $\alpha_{\mathrm{H}(+/ 0)}$ & $\alpha_{\mathrm{H}(0 /-)}$ \\
\hline $\mathrm{NaI}$ & $5.78(0.33)$ & $6.05(0.37)$ & $5.57(0.30)$ & $5.77(0.33)$ \\
$\mathrm{LiI}$ & $5.90(0.26)$ & $6.28(0.32)$ & $5.88(0.26)$ & $5.95(0.27)$ \\
$\mathrm{NaBr}$ & $7.18(0.39)$ & $7.33(0.41)$ & $6.94(0.36)$ & $7.10(0.38)$ \\
$\mathrm{LiBr}$ & $7.40(0.32)$ & $7.78(0.37)$ & $7.40(0.32)$ & $7.40(0.32)$ \\
$\mathrm{NaCl}$ & $8.62(0.45)$ & $8.71(0.46)$ & $8.36(0.42)$ & $8.27(0.41)$ \\
$\mathrm{LiCl}$ & $9.32(0.36)$ & $9.67(0.40)$ & $9.32(0.36)$ & $9.32(0.36)$ \\
$\mathrm{NaF}$ & $12.89(0.59)$ & $12.00(0.51)$ & $12.40(0.55)$ & $12.40(0.55)$ \\
$\mathrm{LiF}$ & $14.54(0.51)$ & $14.05(0.47)$ & $14.41(0.50)$ & $13.92(0.46)$ \\
$\mathrm{MAE}$ & 0.23 & 0.25 & 0.20 & 0.20 \\
\hline \hline
\end{tabular}
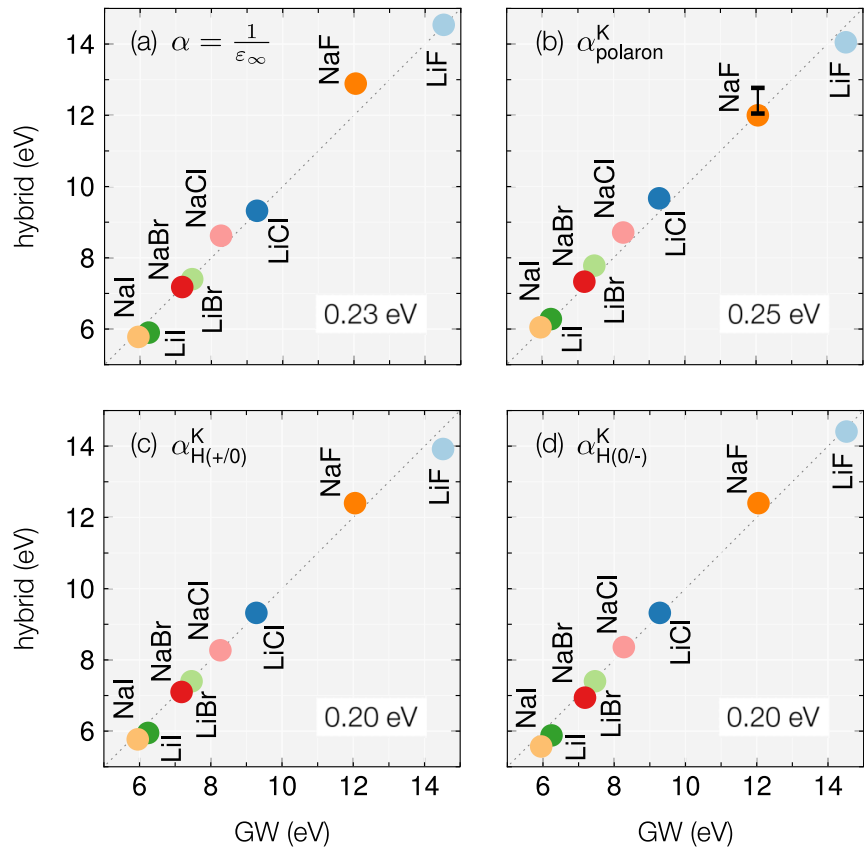

FIG. 1. Band gaps of various alkali halides calculated with the hybrid functional $\operatorname{PBE} 0(\alpha)$ vs reference band gaps obtained at the QS $G \tilde{W}$ level. The fraction of Fock exchange $\alpha$ is set according to different schemes designed (a) to reproduce the long-range screening ( $\alpha=1 / \varepsilon_{\infty}$ ), or to enforce the generalized Koopmans' condition (b) for the $+/ 0$ transition of the polaronic defect, (c) for the $+/ 0$ and (d) $0 /-$ transitions of an interstitial hydrogen impurity. The error bar for $\mathrm{NaF}$ in (b) results from the consideration of a set of defects. MAEs are given in the bottom right corner of each panel.

The agreement is excellent with a mean average error (MAE) of $0.23 \mathrm{eV}$, similar to the MAE obtained when comparing the QS $G \tilde{W}$ band gaps with experiment (cf. Table I). It is clear that for these ionic compounds reproducing the proper long-range screening is sufficient to achieve accurate band gaps.

Next, we consider the generalized Koopmans' condition to determine the free parameter $\alpha$ in the hybrid functional. The Koopmans' condition is an exact physical constraint by which the total energy shows a piece-wise linear dependence upon addition of electrons [3,4]. As the derivative of the energy with respect to the occupation number gives the eigenvalue [66], this condition implies that the single-particle energy level does not shift upon occupation. Hence, the ionization potential of the system with $N$ electrons is thereby identical to the electron affinity of the system with $N-1$ electrons. To exploit this property, it is necessary to focus on localized electronic states.

In this respect, the alkali halides under consideration in this work show spontaneous hole localization upon removal of one electron. This charge localization induces a substantial lattice distortion due to the dimerization of two nearest-neighbor anions, the so-called $V_{K}$ center [55,67]. A semilocal functional does not give any distortion of the bulk lattice due to the SIE, which opposes charge localization. At variance, the use of the hybrid functional PBE0(0.45) ensures the dimerization in all cases. We used the structural configurations obtained in this way without considering any further relaxation for enforcing the Koopmans' condition. Thus, we use the polaronic defect 


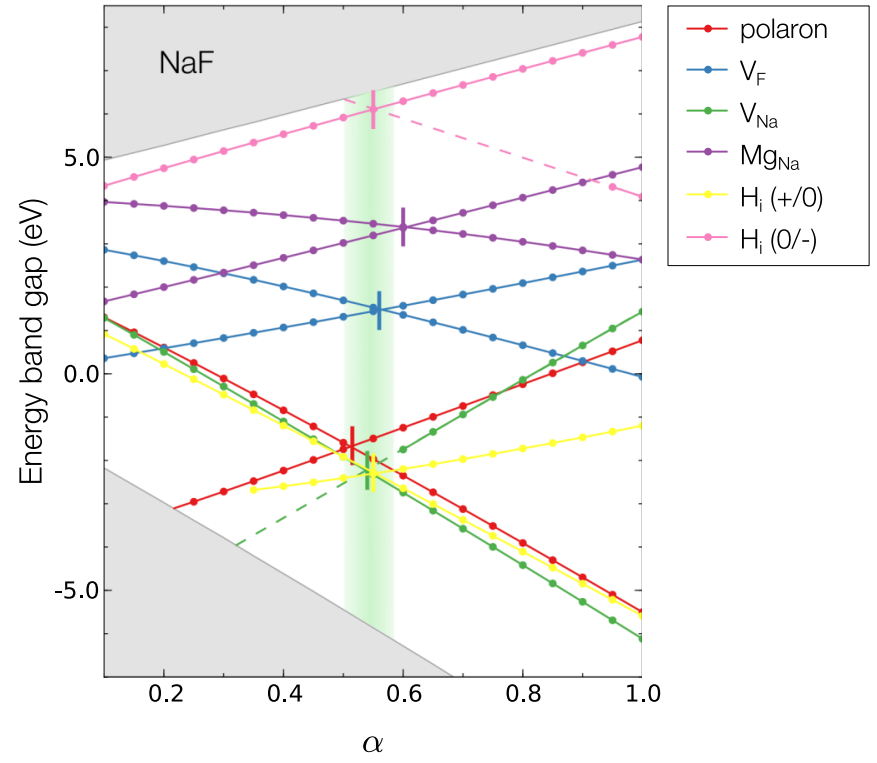

FIG. 2. Band edges and single-particle defect energy levels of various defect states in $\mathrm{NaF}$ vs the fraction of Fock exchange $\alpha$ used in the $\operatorname{PBE} 0(\alpha)$ calculations. The green region is centered at the average $\alpha$ achieved through the Koopmans' condition and its width reflects the standard deviation. The dashed lines are extrapolations of defect energy levels to regions where the defect charge is not well localized.

structure to determine the optimal value of $\alpha$ to be used in the $\operatorname{PBE} 0(\alpha)$ functional.

For all alkali halides studied here, we derive for various values of $\alpha$ the energy levels of the lowest unoccupied state in the positive charge state of the polaron and that of the highest occupied state in the neutral charge state upon vertical electron addition. These energy levels are found to depend linearly on $\alpha$ and their crossing determines $\alpha_{\text {polaron, for which the }}$ Koopmans' condition is satisfied. This procedure is illustrated for $\mathrm{NaF}$ in Fig. 2. The band gaps obtained with $\operatorname{PBE0}\left(\alpha_{\text {polaron }}\right)$ for the present set of alkali halides are given in Table II and are compared to the QSG $\tilde{W}$ band gaps in Fig. 1(b). The agreement is remarkable with mean absolute and mean relative errors of $0.25 \mathrm{eV}$ and $2.7 \%$, respectively. Hence, for the present class of compounds, the band gaps achieved by enforcing the Koopmans' condition agree with those achieved by enforcing the correct long-range screening. The overall accuracy is again comparable to that of QS $G \tilde{W}$ calculations. The present results clearly suggest a strong connection between long-range screening and SIE at the polaronic defect.

Following this suggestion, we infer that the optimal hybrid functional identified via a defect probe should not depend on the nature of the defect, but should directly stem from the longrange screening of the host material. To verify this assumption, we investigate a larger set of defects in the case of $\mathrm{NaF}$. In the same way as for the polaron, we evaluate the energy levels for the $0 /-$ transition of the anion vacancy, the $+/ 0$ transition of the cation vacancy, the $+/ 0$ transition of the $\mathrm{Mg}$ substitutional to $\mathrm{Na}$, and both the $+/ 0$ and $0 /-$ transitions for the hydrogen interstitial $\mathrm{H}_{\mathrm{i}}$. In most cases, the defect states are well localized and the energy levels can be determined for all values of $\alpha$. However, for the highest occupied state of $\mathrm{H}_{\mathrm{i}}$ in the charge state -1 and the lowest unoccupied state in the neutral $\mathrm{Na}$ vacancy, we use linear extrapolations from $\alpha$ regions in which the defect charge is well localized. The results are illustrated in Fig. 2. The values of $\alpha$ satisfying the Koopmans' condition fall in the range $\alpha=0.55 \pm 0.03$. These values give band gaps for $\mathrm{NaF}$ within the interval $12.41 \pm 0.36 \mathrm{eV}$ [cf. error bar in Fig. 1(b)]. The average band gap agrees well with the QS $G \tilde{W}$ reference, showing a deviation of only $3 \%$.

The observed spread in $\alpha$ could result from intrinsic limitations of $\operatorname{PBE} 0(\alpha)$ functionals and/or from inaccuracies due to our modeling procedure [65]. We note that such errors have larger consequences when the slopes of the energy levels vs $\alpha$ in Fig. 2 are less pronounced, such as for the Mg substitutional impurity. In this respect, the interstitial hydrogen is found to give slopes among the strongest. It is thus particularly suggestive to use such a probe for determining the band gap. Figure 2 shows that both the $+/ 0$ and $0 /-$ transitions of $\mathrm{H}_{\mathrm{i}}$ produce a value of $\alpha=0.55$, and thus the same band gap of $12.40 \mathrm{eV}$, in good agreement with the QSG $\tilde{W}$ value of $12.05 \mathrm{eV}$ (cf. Table II). To deepen this relationship, we use the $\mathrm{H}_{\mathrm{i}}$ impurity as a probe to select the optimal hybrid functional also for all the other alkali halides. We use both the $+/ 0$ and $0 /-$ transitions. The obtained band gaps are given in Table II and are compared to the QSG $\tilde{W}$ band gaps in Figs. 1(c) and 1(d), respectively. For all compounds, the two values of $\alpha$ obtained with $\mathrm{H}_{\mathrm{i}}$ almost coincide, as seen for $\mathrm{NaF}$ (cf. Table II). Furthermore, the comparison with the QS $G \tilde{W}$ band gaps yields MAEs as small as $0.20 \mathrm{eV}$, indicating an even closer agreement than obtained with $\alpha=1 / \varepsilon_{\infty}$ or with $\alpha_{\text {polaron }}$.

For the values of $\alpha$ obtained by enforcing either the longrange screening or the Koopmans' condition on defect levels (cf. Table II), we verify that the polaronic distortion is always stabilized. In particular, the contractions of the halide-halide lengths are found in agreement with previous self-interactioncorrected calculations [55]. Hence, the nonempirical hybrid functionals constructed in this work not only yield accurate band gaps, but also correctly feature the formation of small polarons.

To further challenge our approach, we consider a wider class of materials with different bonding character (cf. Table III).

TABLE III. Band gaps $E_{\mathrm{g}}$ obtained with the $\operatorname{PBEO}(\alpha)$ hybrid functional in which the fraction of Fock exchange is set through the enforcement of the Koopmans' condition on the $+/ 0$ transition of the hydrogen interstitial defect $\left(\alpha_{\mathrm{H}(+/ 0)}\right)$ for a set of materials, compared to $\mathrm{QS} G \tilde{W}$ reference values.

\begin{tabular}{lccc}
\hline \hline & $\alpha_{\mathrm{H}(+/ 0)}$ & $E_{\mathrm{g}}^{\mathrm{H}(+/ 0)}(\mathrm{eV})$ & $E_{\mathrm{g}}^{\mathrm{QS} G \tilde{W}}(\mathrm{eV})$ \\
\hline $\mathrm{Si}$ & 0.19 & 1.56 & 1.39 \\
$3 \mathrm{C}-\mathrm{SiC}$ & 0.17 & 2.40 & 2.69 \\
$\mathrm{C}$ & 0.20 & 5.70 & 5.89 \\
$\mathrm{~m}-\mathrm{HfO}_{2}$ & 0.28 & 6.29 & 6.20 \\
$\mathrm{CaO}$ & 0.28 & 6.31 & 6.80 \\
$\mathrm{AlN}$ & 0.26 & 7.30 & 7.53 \\
$\mathrm{MgO}$ & 0.34 & 7.71 & 8.10 \\
$\mathrm{SiO}$ & 0.44 & 10.20 & 10.10 \\
$\mathrm{Ar}$ & 0.60 & 14.60 & 14.62 \\
$\mathrm{MAE}$ & & 0.22 & \\
\hline \hline
\end{tabular}




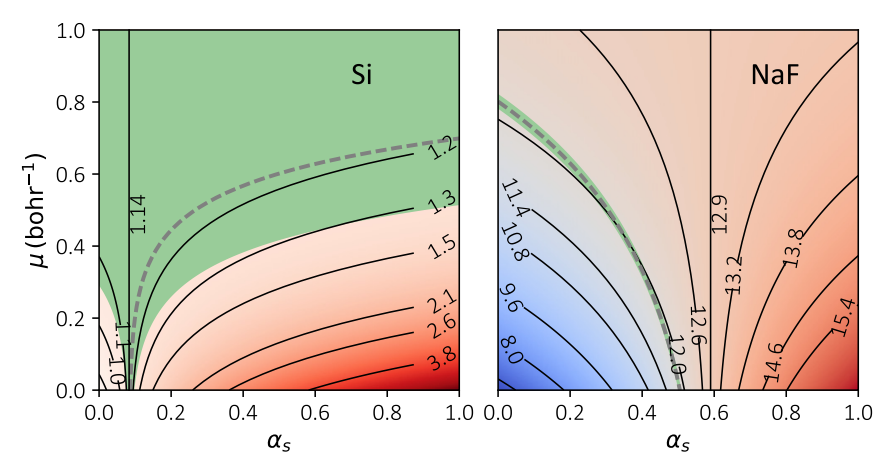

FIG. 3. Isocontour plots of the band gaps of $\mathrm{Si}$ and $\mathrm{NaF}$ in the twodimensional space given by the short-range fraction of Fock exchange $\alpha_{\mathrm{s}}$ and the inverse screening length $\mu$. The band gaps are calculated with a CAM-type hybrid functional, in which the long-range fraction of Fock exchange is set to $\alpha_{\ell}=1 / \varepsilon_{\infty}$. Isovalues of the band gap are shown by solid lines. The dashed lines indicate compliance with the Koopmans' condition, and the green regions deviations below $0.1 \mathrm{eV}$.

We limit our investigation to the $+/ 0$ transition of the $\mathrm{H}_{\mathrm{i}}$ inserted in the largest interatomic void of the solid, as this defect generally gives well localized states. We calculate the band gap with the hybrid functional $\operatorname{PBEO}(\alpha)$, in which $\alpha$ is set to satisfy the Koopmans' condition [65]. The comparison with the QSG $\tilde{W}$ reference values yields a MAE of $0.22 \mathrm{eV}$, close to those found for the alkali halides in Table II. These results further strengthen the connection that we put forward between the band gap and the SIE.

It is of interest to explore hybrid functionals with more free parameters than available in the class of $\operatorname{PBE} 0(\alpha)$, in order to take advantage of the larger flexibility. Therefore, we consider range-separated hybrid functionals $\operatorname{PBE} 0\left(\alpha_{\mathrm{s}}, \alpha_{\ell}, \mu\right)$ following the Coulomb-attenuating method (CAM) of Yanai et al. [24]. This class of functionals includes different fractions of Fock exchange at short $\left(\alpha_{\mathrm{s}}\right)$ and long range $\left(\alpha_{\ell}\right)$, together with the parameter $\mu$, corresponding to the inverse of the screening length over which the range separation occurs. This broader class of CAM-type hybrid functionals englobes the $\operatorname{PBEO}(\alpha)$ functionals as a subclass, as can be seen by taking $\alpha_{\mathrm{s}}=\alpha_{\ell}=\alpha$, but also other standard functionals, such as the HSE functional [23], by setting $\alpha_{\mathrm{s}}=0.25, \alpha_{\ell}=0$, and $\mu=0.106 \mathrm{bohr}^{-1}$. To focus the discussion, we set in the following $\alpha_{\ell}=1 / \varepsilon_{\infty}$, so that we effectively only keep two free parameters, $\alpha_{\mathrm{s}}$ and $\mu$.

Taking $\mathrm{Si}$ and $\mathrm{NaF}$ as representive materials of different bonding character, we systematically vary the parameters $\alpha_{\mathrm{s}}$ and $\mu$, and determine the band gap with the respective CAMtype hybrid functionals. The calculated band gaps are displayed as isocontours in the two-dimensional $\left(\alpha_{\mathrm{s}}, \mu\right)$ space (cf. Fig. 3). In this representation, results corresponding to $\operatorname{PBE} 0\left(\alpha_{\mathrm{s}}\right)$ are recovered for $\mu=0$. The plots also show the curves where the generalized Koopmans' condition is satisfied for $\mathrm{H}_{\mathrm{i}}$ in $\mathrm{Si}$ and for the polaron in NaF. These curves closely follow the evolution of the band-gap isolines. Hence, the band gaps implied by the Koopmans' condition remain constant when more free parameters are considered in the hybrid functional. In the case of $\mathrm{NaF}$, we also verify that all the considered CAM-type hybrid functionals satisfying the Koopmans' condition entail the occurrence of small polarons, with F-F bond lengths remaining identical within $0.01 \AA$. These results demonstrate the robustness of the achieved description. In turn, they suggest that the extension to range-separated hybrid functionals does not lead to any improvement with respect to the $\operatorname{PBE} 0(\alpha)$ functional form.

In conclusion, we demonstrate a close connection between the band gap, the long-range screening, and the self-interaction error. The same fraction of Fock exchange in one-parameter hybrid functionals is found to be uniquely determined by any of these three properties. These results reveal a general route for achieving accurate band gaps in a nonempirical fashion and without resorting to computationally demanding $G W$ calculations, for instance through the use of simple hydrogen probes.

We thank A. Baldereschi for useful discussions. This work is supported by the Swiss National Science Foundation (SNSF) under Grant No. 200020-172524. The calculations have been performed at the Swiss National Supercomputing Center (CSCS) and at the EPFL.
[1] L. J. Sham and M. Schlüter, Phys. Rev. Lett. 51, 1888 (1983).

[2] J. P. Perdew and A. Zunger, Phys. Rev. B 23, 5048 (1981).

[3] J. P. Perdew, R. G. Parr, M. Levy, and J. L. Balduz, Phys. Rev. Lett. 49, 1691 (1982).

[4] P. Mori-Sánchez, A. J. Cohen, and W. Yang, J. Chem. Phys. 125, 201102 (2006).

[5] G. Pacchioni, F. Frigoli, D. Ricci, and J. A. Weil, Phys. Rev. B 63, 054102 (2000).

[6] J. Lægsgaard and K. Stokbro, Phys. Rev. Lett. 86, 2834 (2001).

[7] A. Filippetti and N. A. Spaldin, Phys. Rev. B 67, 125109 (2003).

[8] P. Mori-Sánchez, A. J. Cohen, and W. Yang, Phys. Rev. Lett. 100, 146401 (2008).

[9] X. Zheng, A. J. Cohen, P. Mori-Sánchez, X. Hu, and W. Yang, Phys. Rev. Lett. 107, 026403 (2011).

[10] J. Ma and L.-W. Wang, Sci. Rep. 6, 24924 (2016).
[11] P. Deák, Q. Duy Ho, F. Seemann, B. Aradi, M. Lorke, and T. Frauenheim, Phys. Rev. B 95, 075208 (2017).

[12] M. Weng, S. Li, J. Ma, J. Zheng, F. Pan, and L.-W. Wang, Appl. Phys. Lett. 111, 054101 (2017).

[13] N. L. Nguyen, N. Colonna, A. Ferretti, and N. Marzari, arXiv:1708.08518.

[14] L. Hedin, Phys. Rev. 139, A796 (1965).

[15] M. S. Hybertsen and S. G. Louie, Phys. Rev. B 34, 5390 (1986).

[16] M. Shishkin, M. Marsman, and G. Kresse, Phys. Rev. Lett. 99, 246403 (2007).

[17] W. Chen and A. Pasquarello, Phys. Rev. B 92, 041115 (2015).

[18] A. Alkauskas, P. Broqvist, and A. Pasquarello, Phys. Status Solidi B 248, 775 (2011).

[19] A. Carvalho, A. Alkauskas, A. Pasquarello, A. K. Tagantsev, and N. Setter, Phys. Rev. B 80, 195205 (2009). 
[20] J. L. Lyons, A. Janotti, and C. G. Van de Walle, Phys. Rev. Lett. 108, 156403 (2012).

[21] G. Miceli and A. Pasquarello, Phys. Rev. B 93, 165207 (2016).

[22] J. P. Perdew, M. Ernzerhof, and K. Burke, J. Chem. Phys. 105, 9982 (1996).

[23] J. Heyd, G. E. Scuseria, and M. Ernzerhof, J. Chem. Phys. 118, 8207 (2003); 124, 219906 (2006).

[24] T. Yanai, D. P. Tew, and N. C. Handy, Chem. Phys. Lett. 393, 51 (2004).

[25] F. Gygi and A. Baldereschi, Phys. Rev. Lett. 62, 2160 (1989).

[26] D. M. Bylander and L. Kleinman, Phys. Rev. B 41, 7868 (1990).

[27] V. Fiorentini and A. Baldereschi, Phys. Rev. B 51, 17196 (1995).

[28] T. Shimazaki and Y. Asai, Chem. Phys. Lett. 466, 91 (2008).

[29] T. Shimazaki and Y. Asai, J. Chem. Phys. 130, 164702 (2009).

[30] T. Shimazaki and Y. Asai, J. Chem. Phys. 132, 224105 (2010).

[31] M. A. L. Marques, J. Vidal, M. J. T. Oliveira, L. Reining, and S. Botti, Phys. Rev. B 83, 035119 (2011).

[32] D. Koller, P. Blaha, and F. Tran, J. Phys.: Condens. Matter 25, 435503 (2013).

[33] J. H. Skone, M. Govoni, and G. Galli, Phys. Rev. B 89, 195112 (2014).

[34] M. Gerosa, C. E. Bottani, L. Caramella, G. Onida, C. Di Valentin, and G. Pacchioni, Phys. Rev. B 91, 155201 (2015).

[35] J. H. Skone, M. Govoni, and G. Galli, Phys. Rev. B 93, 235106 (2016).

[36] A. P. Gaiduk, M. Govoni, R. Seidel, J. H. Skone, B. Winter, and G. Galli, J. Am. Chem. Soc. 138, 6912 (2016).

[37] N. P. Brawand, M. Vörös, M. Govoni, and G. Galli, Phys. Rev. X 6, 041002 (2016).

[38] M. Gerosa, C. Di Valentin, C. E. Bottani, G. Onida, and G. Pacchioni, J. Chem. Phys. 143, 111103 (2015).

[39] A. J. Cohen, P. Mori-Sánchez, and W. Yang, J. Chem. Phys. 126, 191109 (2007).

[40] I. Dabo, A. Ferretti, N. Poilvert, Y. Li, N. Marzari, and M. Cococcioni, Phys. Rev. B 82, 115121 (2010).

[41] T. Stein, H. Eisenberg, L. Kronik, and R. Baer, Phys. Rev. Lett. 105, 266802 (2010).

[42] S. Refaely-Abramson, R. Baer, and L. Kronik, Phys. Rev. B 84, 075144 (2011).

[43] L. Kronik, T. Stein, S. Refaely-Abramson, and R. Baer, J. Chem. Theory Comput. 8, 1515 (2012).

[44] S. Refaely-Abramson, S. Sharifzadeh, N. Govind, J. Autschbach, J. B. Neaton, R. Baer, and L. Kronik, Phys. Rev. Lett. 109, 226405 (2012).
[45] J. Autschbach and M. Srebro, Acc. Chem. Res. 47, 2592 (2014).

[46] H. Phillips, Z. Zheng, E. Geva, and B. D. Dunietz, Org. Electron. 15, 1509 (2014).

[47] M. E. Foster, J. D. Azoulay, B. M. Wong, and M. D. Allendorf, Chem. Sci. 5, 2081 (2014).

[48] T. Körzdörfer and J.-L. Brédas, Acc. Chem. Res. 47, 3284 (2014).

[49] V. Atalla, I. Y. Zhang, O. T. Hofmann, X. Ren, P. Rinke, and M. Scheffler, Phys. Rev. B 94, 035140 (2016).

[50] N. Sai, P. F. Barbara, and K. Leung, Phys. Rev. Lett. 106, 226403 (2011).

[51] S. Refaely-Abramson, S. Sharifzadeh, M. Jain, R. Baer, J. B. Neaton, and L. Kronik, Phys. Rev. B 88, 081204 (2013).

[52] D. A. Egger, S. Weissman, S. Refaely-Abramson, S. Sharifzadeh, M. Dauth, R. Baer, S. Kümmel, J. B. Neaton, E. Zojer, and L. Kronik, J. Chem. Theory Comput. 10, 1934 (2014).

[53] N. L. Nguyen, G. Borghi, A. Ferretti, I. Dabo, and N. Marzari, Phys. Rev. Lett. 114, 166405 (2015).

[54] S. Lany and A. Zunger, Phys. Rev. B 81, 205209 (2010).

[55] B. Sadigh, P. Erhart, and D. Åberg, Phys. Rev. B 92, 075202 (2015).

[56] F. C. Brown, C. Gähwiller, H. Fujita, A. B. Kunz, W. Scheifley, and N. Carrera, Phys. Rev. B 2, 2126 (1970).

[57] H. Li, J.Phys. Chem. Ref. Data 5, 329 (1976).

[58] W. Chen and A. Pasquarello, Phys. Rev. B 88, 115104 (2013).

[59] C. Freysoldt, J. Neugebauer, and C. G. Van de Walle, Phys. Rev. Lett. 102, 016402 (2009).

[60] H.-P. Komsa, T. T. Rantala, and A. Pasquarello, Phys. Rev. B 86, 045112 (2012).

[61] W. Chen and A. Pasquarello, J. Phys.: Condens. Matter 27, 133202 (2015).

[62] J. P. Perdew, K. Burke, and M. Ernzerhof, Phys. Rev. Lett. 77, 3865 (1996).

[63] S. V. Faleev, M. van Schilfgaarde, and T. Kotani, Phys. Rev. Lett. 93, 126406 (2004).

[64] M. van Schilfgaarde, T. Kotani, and S. Faleev, Phys. Rev. Lett. 96, 226402 (2006).

[65] See Supplemental Material at http://link.aps.org/supplemental/ 10.1103/PhysRevB.97.121112 for the structural parameters and the computational details used in the hybrid and QS $G \tilde{W}$ calculations, together with the dielectric constants used in the finite-size corrections.

[66] J. F. Janak, Phys. Rev. B 18, 7165 (1978).

[67] W. Känzig, Phys. Rev. 99, 1890 (1955). 